\title{
Full Control of the Spin-Wave Damping in a Magnetic Insulator Using Spin-Orbit Torque
}

\author{
A. Hamadeh, ${ }^{1}$ O. d'Allivy Kelly, ${ }^{2}$ C. Hahn, ${ }^{1}$ H. Meley, ${ }^{1}$ R. Bernard, ${ }^{2}$ A. H. Molpeceres, ${ }^{2}$ V. V. Naletov, ${ }^{1,2,3}$ M. Viret, \\ A. Anane, ${ }^{2}$ V. Cros,${ }^{2}$ S. O. Demokritov, ${ }^{4,8}$ J. L. Prieto, ${ }^{5}$ M. Muñoz ${ }^{6}$ G. de Loubens, ${ }^{1, \dagger}$ and O. Klein ${ }^{1,7, *}$ \\ ${ }^{1}$ Service de Physique de l'État Condensé (CNRS URA 2464), CEA Saclay, 91191 Gif-sur-Yvette, France \\ ${ }^{2}$ Unité Mixte de Physique CNRS/Thales and Université Paris Sud 11, 1 avenue Fresnel, 91767 Palaiseau, France \\ ${ }^{3}$ Institute of Physics, Kazan Federal University, Kazan 420008, Russian Federation \\ ${ }^{4}$ Department of Physics, University of Muenster, 48149 Muenster, Germany \\ ${ }^{5}$ Instituto de Sistemas Optoelectrónicos y Microtecnología (UPM), Madrid 28040, Spain \\ ${ }^{6}$ Instituto de Microelectrónica de Madrid (CNM, CSIC), Madrid 28760, Spain \\ ${ }^{7}$ SPINTEC, UMR CEA/CNRS/UJF-Grenoble 1/Grenoble-INP, INAC, 38054 Grenoble, France \\ ${ }^{8}$ Institute of Metal Physics, Ural Division of RAS, Yekaterinburg 620041, Russia
}

(Received 28 May 2014; published 7 November 2014)

\begin{abstract}
It is demonstrated that the threshold current for damping compensation can be reached in a $5 \mu \mathrm{m}$ diameter $\mathrm{YIG}(20 \mathrm{~nm}) \mid \mathrm{Pt}(7 \mathrm{~nm})$ disk. The demonstration rests upon the measurement of the ferromagnetic resonance linewidth as a function of $I_{\mathrm{dc}}$ using a magnetic resonance force microscope (MRFM). It is shown that the magnetic losses of spin-wave modes existing in the magnetic insulator can be reduced or enhanced by at least a factor of 5 depending on the polarity and intensity of an in-plane dc current $I_{\mathrm{dc}}$ flowing through the adjacent normal metal with strong spin-orbit interaction. Complete compensation of the damping of the fundamental mode by spin-orbit torque is reached for a current density of $\sim 3 \times 10^{11} \mathrm{~A} \cdot \mathrm{m}^{-2}$, in agreement with theoretical predictions. At this critical threshold the MRFM detects a small change of static magnetization, a behavior consistent with the onset of an auto-oscillation regime.
\end{abstract}

DOI: 10.1103/PhysRevLett.113.197203

PACS numbers: 85.75.-d, 76.50.+g

The spin-orbit interaction (SOI) [1-3] has been recently shown to be an interesting and useful addition in the field of spintronics. This subject capitalizes on adjoining a strong SOI normal metal next to a thin magnetic layer [4]. The SOI converts a charge current $J_{c}$ into a spin current $J_{s}$ with an efficiency parametrized by $\Theta_{\mathrm{SH}}$, the spin Hall angle $[5,6]$. Recently, it was demonstrated experimentally that the spin current produced in this way can switch the magnetization in a dot $[7,8]$ or can partially compensate the damping [9-11], allowing the lifetime of propagating spin waves [12] to be increased beyond their natural decay time $\tau$. These two effects open potential applications in storage devices and in microwave signal processing.

The effect is based on the fact that the spin current $J_{s}$ exerts a torque on the magnetization, corresponding to an additional damping term $\Gamma_{s}=\gamma J_{s} /\left(t_{\mathrm{FM}} M_{s}\right)$, where $t_{\mathrm{FM}}$ is the thickness of the magnetic layer, $M_{s}$ is its spontaneous magnetization, and $\gamma$ is the gyromagnetic ratio. In the case of metallic ferromagnets [13-15], it was established that $\Gamma_{s}$ can fully compensate the natural damping $1 / \tau$ at a critical spin current $J_{s}^{*}$, which determines the onset of autooscillation of the magnetization:

$$
J_{s}^{*}=-\frac{1}{\tau} \frac{t_{\mathrm{FM}} M_{s}}{\gamma} .
$$

An important benefit of the SOI is that $J_{c}$ and $J_{s}$ are linked through a cross product, allowing a charge current flowing in plane to produce a spin current flowing out of plane.
Hence, it enables the transfer of spin angular momentum to nonmetallic materials and in particular to insulating oxides, which offer improved performance compared to their metallic counterparts. Among all oxides, yttrium iron garnet (YIG) holds a special place for having the lowest known spin-wave (SW) damping factor. In 2010, Kajiwara et al. reported on the efficient transmission of spin current through the YIG|Pt interface [16]. It was shown that $J_{s}$ produced by the excitation of ferromagnetic resonance (FMR) in YIG can cross the YIG|Pt interface and be converted into $J_{c}$ in Pt through the inverse spin Hall effect (ISHE). This finding was reproduced in numerous experimental works [17-23]. In the same paper [16], the reciprocal effect $[24,25]$ was also reported as $J_{s}$ produced in Pt by the direct spin Hall effect could be transferred to the $1.3 \mu \mathrm{m}$ thick YIG, resulting in damping compensation [16]. However, attempts to directly measure the expected change of the resonance linewidth of YIG as a function of the dc current have so far failed.[21,22] This is raising fundamental questions about the reciprocity of the spin transparency $T$ of the interface between a metal and a magnetic insulator. This coefficient enters in the ratio between $J_{c}$ in $\mathrm{Pt}$ and $J_{s}$ in YIG through

$$
J_{s}=T \Theta_{\mathrm{SH}} \frac{\hbar}{2 e} J_{c},
$$

where $e$ is the electron charge and $\hbar$ the reduced Planck constant. $T$ depends on the transport characteristics of the normal metal as well as on the spin-mixing conductance 
$G_{\uparrow \downarrow}$, which parametrizes the scattering of the spin angular momentum at the YIG|Pt interface [26].

At the heart of this debate lies the exact value of the threshold current [27]. The lack of visible effects reported in Refs. [21,22], although inconsistent with Ref. [16], is coherent with the estimation of the threshold current density of a few $10^{12} \mathrm{~A} \cdot \mathrm{m}^{-2}$ using Eqs. (1) and (2) and typical parameters for the materials [28]. This theoretical current density is at least 1 order of magnitude larger than the maximum $J_{c}$ that could be injected in the Pt so far. Importantly, the previous reported experiments were performed on large (millimeter sized) structures, where many nearly degenerate SW modes compete for feeding from the same dc source, a phenomenon that could become selflimiting and prevent the onset of auto-oscillations [11]. To isolate a single candidate mode, we have recently reduced the lateral dimensions of the YIG pattern, as quantization results in increased frequency gaps between the dynamical modes [29]. This requires us to grow very thin films of high quality YIG [23,30-32]. Benefiting from our progress in the epitaxial growth of YIG films by pulsed laser deposition [22], we propose to study the FMR linewidth as a function of the dc current in a micron-size YIG|Pt disk [29].

Figure 1 shows a schematic of the experimental setup. A YIG|Pt disk $5 \mu \mathrm{m}$ in diameter is connected to two $\mathrm{Au}$ contact electrodes $1.5 \mu \mathrm{m}$ apart (see the microscopy image) across which a positive voltage generates a current flow $J_{c}$

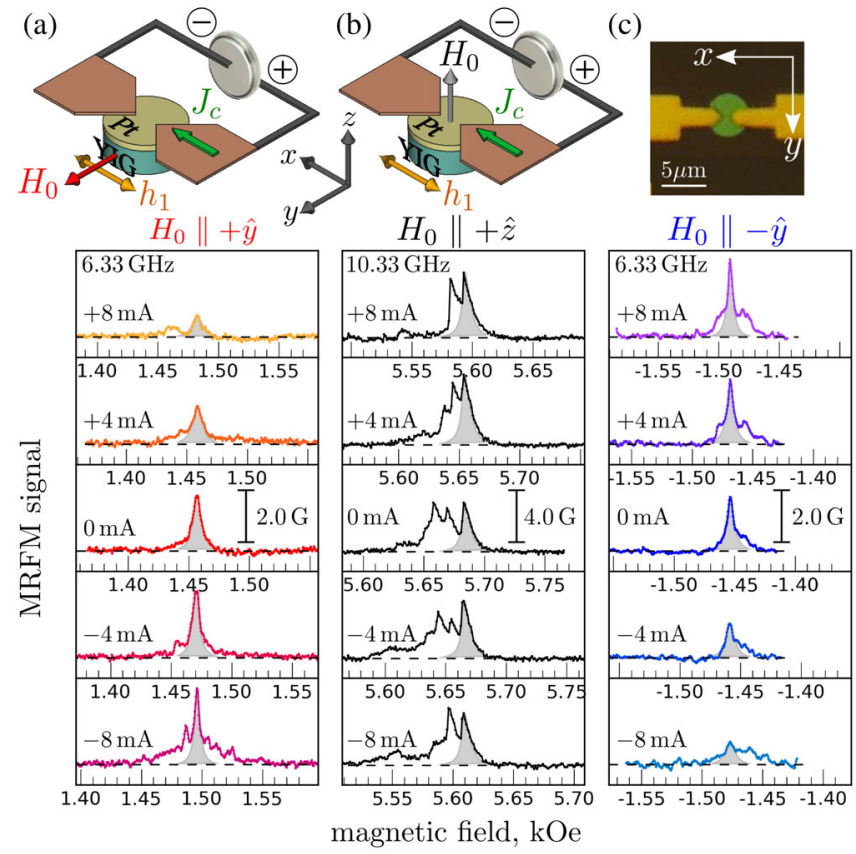

FIG. 1 (color online). MRFM spectra of the YIG|Pt microdisk as a function of current for different field orientations: (a) $H_{0} \|+\hat{y}$ at $f=6.33 \mathrm{GHz}$ (red tone), (b) $H_{0} \|+\hat{z}$ at $f=10.33 \mathrm{GHz}$ (black), (c) $H_{0} \|-\hat{y}$ at $f=6.33 \mathrm{GHz}$ (blue tone). The highest amplitude mode is used for linewidth analysis (shaded area). Field axes are shifted so as to align the peaks vertically. In-plane and out-of-plane field orientations are sketched above. The top right frame is a microscopy image of the sample. along the $+\hat{x}$ direction. The microdisk is patterned out of a $20 \mathrm{~nm}$ thick epitaxial YIG film with a $7 \mathrm{~nm}$ thick Pt layer sputtered on top. The YIG and Pt layers have been fully characterized in previous studies [22,33]. Their characteristics are reported in Table I.

The sample is mounted inside a room temperature magnetic resonance force microscope (MRFM), which detects the SW absorption spectrum mechanically [34-36]. The excitation is provided by a stripline (not shown in the sketches of Fig. 1) generating a linearly polarized microwave field $h_{1}$ along the $\hat{x}$ direction. The detection is based on monitoring the deflection of a mechanical cantilever with a magnetic Fe particle affixed to its tip, coupled dipolarly to the sample. The FMR spectrum is obtained by recording the vibration amplitude of the cantilever while scanning the external bias magnetic field $H_{0}$ at constant microwave excitation frequency $f=\omega /(2 \pi)$. When FMR conditions are met, the precession cone opens up by $\theta$ and in consequence the static magnetization (projection along the precession axis) decreases by $\delta \bar{M}=(1-\cos \theta) M_{s}$, hereby exciting mechanically the cantilever. The MRFM is placed between the poles of an electromagnet, generating a uniform magnetic field $H_{0}$, which can be set along $\hat{y}$ or $\hat{z}$ (i.e., perpendicularly to both $h_{1}$ and $J_{c}$ ).

We start by measuring the effect of a dc current $I_{\mathrm{dc}}$ on the FMR spectra when the disk is magnetized in plane by a magnetic field along the $+\hat{y}$ direction (positive field). The spectra recorded at $f=6.33 \mathrm{GHz}$ are shown in Fig. 1(a) in red tones. The middle row shows the absorption at zero current. The MRFM signal corresponds to a diminution of the static magnetization $4 \pi \delta \bar{M}_{y}$ of about $2 \mathrm{G}$, i.e., a precession cone of $\theta=2.5^{\circ}$. As the the electrical current is varied, we observe very clearly a change of the linewidth. At negative current, the linewidth decreases, to reach about half the initial value at $I_{\mathrm{dc}}=-8 \mathrm{~mA}$. This decrease is strong enough so that the individual modes can be resolved spectroscopically within the main peak. Concomitantly, the amplitude of the MRFM signal increases. The opposite behavior is observed when the current polarity is reversed. At positive current, the linewidth increases to reach about twice the initial value at $I_{\mathrm{dc}}=+8 \mathrm{~mA}$, and the amplitude of the signal decreases.

$I_{\mathrm{dc}}= \pm 12 \mathrm{~mA}$ is the maximum current that we have injected in our sample to avoid irreversible effects. We estimate, from the Pt resistance, the sample temperature to be $90^{\circ} \mathrm{C}$ at the maximum current. This Joule heating

TABLE I. Transport and magnetic properties of the Pt and bare YIG layers, respectively, from Refs. [33] and [22].

\begin{tabular}{ccccc}
\hline \hline $\mathrm{Pt}$ & $t_{\mathrm{Pt}}(\mathrm{nm})$ & $\sigma\left(\Omega^{-1} \cdot \mathrm{m}^{-1}\right)$ & $\lambda_{\mathrm{SD}}(\mathrm{nm})$ & $\Theta_{\mathrm{SH}}$ \\
\hline & 7 & $5.8 \times 10^{6}$ & 3.5 & 0.056 \\
$\mathrm{YIG}$ & $t_{\mathrm{YIG}}(\mathrm{nm})$ & $4 \pi M_{s}(\mathrm{G})$ & $\gamma\left(\mathrm{rad} \cdot \mathrm{s}^{-1} \cdot \mathrm{G}^{-1}\right)$ & $\alpha_{0}$ \\
& 20 & $2.1 \times 10^{3}$ & $1.79 \times 10^{7}$ & $2.3 \times 10^{-4}$ \\
\hline \hline
\end{tabular}


reduces $4 \pi M_{s}$ at a rate of $4.8 \mathrm{G} / \mathrm{K}$, and it results in an even shift of the resonance field towards higher field [37].

In Fig. 1(b), we show the FMR spectra at $f=$ $10.33 \mathrm{GHz}$ in the perpendicular geometry; i.e., $H_{0}$ is along $\hat{z}$. In contrast to the previous case, the linewidth does not change with current. This is expected as no net spin transfer torque is exerted by the spin current on the precessing magnetization in this configuration. Note that due to Joule heating, the spectrum now shifts towards lower field due to the decrease of $M_{s}$ as the current increases.

We now come back to the in-plane geometry, but this time, the magnetic field is reversed compared to Fig. 1(a), i.e., applied along $-\hat{y}$ (negative field). The corresponding spectra are presented in Fig. 1(c) using blue tones. As expected for the symmetry of the direct spin Hall effect, the observed behavior is inverted with respect to Fig. 1(a): a positive (negative) current now reduces (broadens) the linewidth.

We report in Fig. 2 the values of $\Delta H_{\|}$, the full linewidth measured in the in-plane geometry, as a function of current. The data points follow approximately a straight line, whose slope $\pm 0.5 \mathrm{Oe} / \mathrm{mA}$ reverses with the direction of $H_{0}$ along $\pm \hat{y}$ and whose intercept with the abscissa axis occurs at $I_{6.33 \mathrm{GHz}}^{*}=\mp 14 \mathrm{~mA}$. Moreover, we emphasize that the variation of linewidth covers about a factor of 5 on the full range of current explored. For comparison, we have reported on the same figure the values of $\Delta H_{\perp}$ measured at $10.33 \mathrm{GHz}$ (black).

The inset of Fig. 2 shows the inverse spin Hall voltage $V_{\text {ISHE }}$ measured at $I_{\mathrm{dc}}=0 \mathrm{~mA}$ and $f=6.33 \mathrm{GHz}$. This voltage results from the spin current produced by spin pumping from YIG to Pt and its subsequent conversion into charge current by the ISHE [16]. Its sign changes with the direction of the bias magnetic field, as shown by the blue and red $V_{\text {ISHE }}$ spectra. This observation confirms that a spin current can flow from YIG to Pt and that damping reduction occurs for a current polarity corresponding to a negative product of $V_{\mathrm{ISHE}}$ and $I_{\mathrm{dc}}$.

To gain more insight into these results, we now analyze the frequency dependence of the full linewidth at half

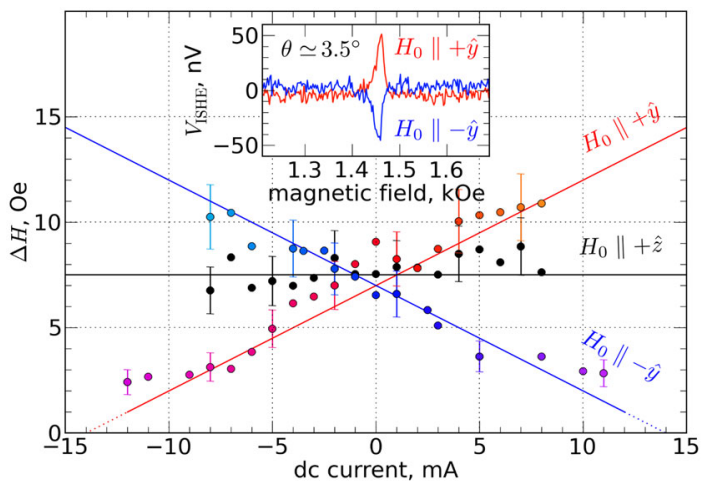

FIG. 2 (color online). Variation of the full linewidth $\Delta H_{\|}$ measured at $6.33 \mathrm{GHz}$ as a function of $I_{\mathrm{dc}}$ for $H_{0} \|+\hat{y}$ (red) and $H_{0} \|-\hat{y}$ (blue). Added to the plot is the measurement of $\Delta H_{\perp}$ measured at $10.33 \mathrm{GHz}$ (black). Inset: detection of $V_{\text {ISHE }}$ as a function of $H_{0}$ at $f=6.33 \mathrm{GHz}$ and $I_{\mathrm{dc}}=0$. maximum for three values of dc current $(0, \pm 6 \mathrm{~mA})$ for both the out-of-plane and in-plane geometries. We start with the out-of-plane data, plotted in Fig. 3(a). The dispersion relation displayed in the inset follows the Kittel law $\omega=\gamma\left(H_{0}-4 \pi N_{\text {eff }} M_{s}\right)$, where $N_{\text {eff }}$ is an effective demagnetizing factor (close to $1[38,39]$ ). The linewidth $\Delta H_{\perp}$ increases linearly with frequency along a line that intercepts the origin, a signature that the resonance is homogeneously broadened [29]. In this geometry, the Gilbert damping coefficient is simply $\alpha=\gamma \Delta H_{\perp} /(2 \omega)=$ $1.1 \times 10^{-3}$ and the relaxation time $\tau=1 /(\alpha \omega)$. We also report on this figure the fact that, at $10.33 \mathrm{GHz}, \Delta H_{\perp}=$ $7 \mathrm{Oe}$ is independent of the current [see also Fig. 1(b)].

The damping found in our YIG|Pt is significantly larger than the one measured in the bare YIG $\alpha_{0}=2.3 \times 10^{-4}$ (cf. Table I). This difference is due to the spin pumping effect, and enables us to determine the spin-mixing conductance of our YIG|Pt interface through $[40,41]$

$$
\alpha=\alpha_{0}+\frac{\gamma \hbar}{4 \pi M_{s} t_{\mathrm{YIG}}} \frac{G_{\uparrow \downarrow}}{G_{0}},
$$

where $G_{0}=2 e^{2} / h$ is the quantum of conductance. The measured increase of almost $9 \times 10^{-4}$ for the damping corresponds to $G_{\uparrow \downarrow}=1.5 \times 10^{14} \Omega^{-1} \cdot \mathrm{m}^{-2}$, in agreement with a previous determination made on similar $\mathrm{YIG} \mid \mathrm{Pt}$ nanodisks [29]. This value allows us to estimate the spin transparency of our interface [26], $T=G_{\uparrow \downarrow} \tanh \left(t_{\mathrm{Pt}} / 2 \lambda_{\mathrm{sd}}\right) /$ $\left[G_{\uparrow \downarrow} \operatorname{coth}\left(t_{\mathrm{Pt}} / \lambda_{\mathrm{sd}}\right)+\sigma /\left(2 \lambda_{\mathrm{sd}}\right)\right] \simeq 0.12$, where $\sigma$ is the $\mathrm{Pt}$ conductivity and $\lambda_{\mathrm{sd}}$ is its spin-diffusion length. Moreover, the spin-mixing conductance can be used to analyze quantitatively the dc ISHE voltage produced at resonance $[21,42,43]$. Using the parameters of Table I and the value of $G_{\uparrow \downarrow}$, we find that the $50 \mathrm{nV}$ voltage measured in the inset of Fig. 2 is produced by an angle of precession $\theta \simeq 3.5^{\circ}$, which lies in the expected range.

We now turn to the in-plane data, presented in Fig. 3(b). The dispersion relation plotted in the inset follows the Kittel law $\omega=\gamma \sqrt{H_{0}\left(H_{0}+4 \pi N_{\text {eff }} M_{s}\right)}$. In this case,

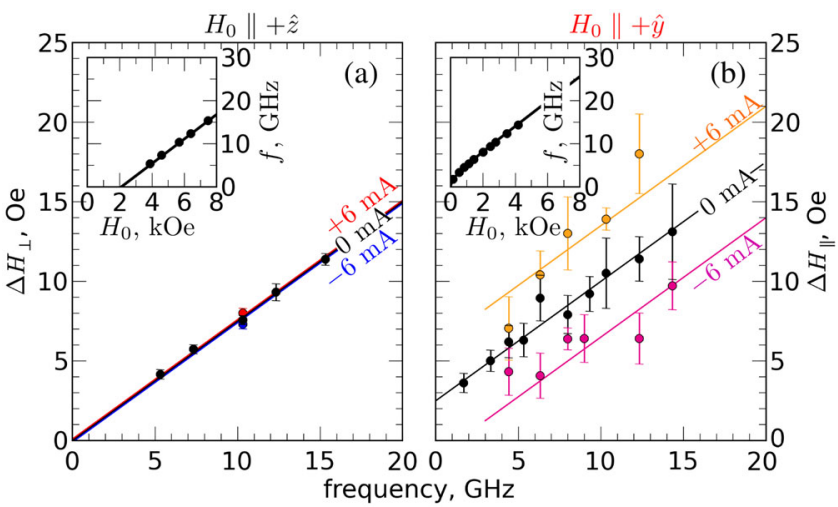

FIG. 3 (color online). Frequency dependence of the linewidth for three values of the $\mathrm{dc}$ current $(0, \pm 6 \mathrm{~mA})$ (a) in the perpendicular geometry and (b) in the parallel geometry. Insets show the corresponding dispersion relations $f\left(H_{0}\right)$. 
$1 / \tau=\alpha\left(\partial \omega / \partial H_{0}\right)(\omega / \gamma)$. For $I_{\mathrm{dc}}=0 \mathrm{~mA}$ the slope of the linewidth versus frequency is exactly the same as that in the perpendicular direction $\alpha=1.1 \times 10^{-3}$. For this geometry, however, the line does not intercept the origin, indicating a finite amount of inhomogeneous broadening $\Delta H_{0}=$ 2.5 Oe, i.e., the presence of several modes within the resonance line. Considering now the effect of the current, we recall that the expression for the linewidth reduces to

$$
\Delta H_{\|}=\Delta H_{0}+2 \alpha \frac{\omega}{\gamma}+2 \frac{J_{s}}{M_{s} t_{\mathrm{YIG}}},
$$

in the high field regime (i.e., when $\partial \omega / \partial H_{0} \simeq \gamma$ ). This expresses the fact that $\Gamma_{s}$, the damping term associated with the transfer of spin to the YIG, comes as an additional contribution to the existing broadening. In Fig. 3(b), we observe that the $0 \mathrm{~mA}$-fit line shifts by \pm 3 Oe when $I_{\mathrm{dc}}$ is set to $\pm 6 \mathrm{~mA}$. This is consistent with the rate of $0.5 \mathrm{Oe} / \mathrm{mA}$ reported at $6.33 \mathrm{GHz}$ in Fig. 2.

In order to investigate the autonomous dynamics of the YIG layer and exceed the compensation current $I^{*}$ we now perform measurements at lower excitation frequency, where the threshold current is estimated below $12 \mathrm{~mA}$. In Fig. 4(a), we present a density plot of the MRFM spectra acquired at $4.33 \mathrm{GHz}$ as a function of the in-plane magnetic field and $I_{\mathrm{dc}}$ through the Pt. The measured signal is clearly asymmetric in $I_{\mathrm{dc}}$. At positive current, it broadens and its amplitude decreases, almost disappearing above $+8 \mathrm{~mA}$, whereas at negative current, it becomes narrower and the amplitude is maximal at $I_{\mathrm{dc}}<-10 \mathrm{~mA}$.

The power integrated over the full field range normalized by its value at $0 \mathrm{~mA}$ and the linewidth variation versus $I_{\mathrm{dc}}$ are plotted in Figs. 4(b) and 4(c), respectively. The normalized integrated power varies by a factor of 5 from +12 to $-12 \mathrm{~mA}$ following an inverse law on $I_{\mathrm{dc}}$ (see continuous line), which is consistent with the spin transfer effect $[11,44]$. The linewidth varies roughly linearly with $I_{\mathrm{dc}}$ : it increases from 6 Oe at $0 \mathrm{~mA}$ up to $14 \mathrm{Oe}$ at $+12 \mathrm{~mA}$ and it reaches a minimum value close to 2 Oe between -8 and $-11 \mathrm{~mA}$. It is interesting to note that this happens in a region of the density plot where the evolution of the signal displays some kind of discontinuity, with the appearance of several high amplitude peaks in the spectrum [see arrow in Fig. 4(a)].

To check if this is the threshold current, we present in Fig. 4(d) results of an experiment where no rf excitation is applied to the system. Here, the dc current is modulated at the MRFM cantilever frequency by $\delta I=0.15 \mathrm{~mA}_{\mathrm{pp}}$ and the induced $\delta \bar{M}_{y}$ is probed as a function of $I_{\mathrm{dc}}$. This experiment thus provides a differential measurement $d \bar{M}_{y} / d I_{\mathrm{dc}}$ of the magnetization (in analogy with $d V / d I$ measurements in transport experiments). At $H_{0}=$ $0.92 \mathrm{kOe}$, a peak in $d \bar{M}_{y} / d I_{\mathrm{dc}}$ is measured around $-9 \mathrm{~mA}$, suggesting auto-oscillations in the YIG layer. It corresponds to a variation of $4 \pi \delta \bar{M}_{y} \simeq 0.5 \mathrm{G}$, i.e., a change of the angle of precession by $1.3^{\circ}$ induced by the modulation of current. Moreover, this narrow peak observed in $d \bar{M}_{y} / d I_{\mathrm{dc}}$ shifts linearly in dc current with the applied

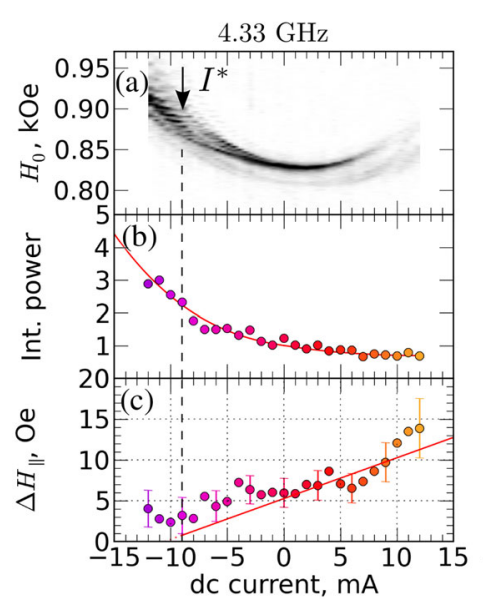

$4 \pi \partial M_{z} / \partial I_{\mathrm{dc}}, 1 \mathrm{G} / \mathrm{mA}$ per div

FIG. 4 (color online). (a) Density plot of the MRFM spectra at $4.33 \mathrm{GHz}$ versus field and current $I_{\mathrm{dc}} \in[-12,+12] \mathrm{mA}$. The color scale represents $4 \pi \delta \bar{M}_{y}$ (white: $0 \mathrm{G}$, black: $1.5 \mathrm{G}$ ). (b) Evolution of integrated power versus $I_{\mathrm{dc}}$. (c) Dependence of linewidth on $I_{\mathrm{dc}}$. (d) Differential measurements of $\bar{M}_{y}\left(I_{\mathrm{dc}}\right.$ modulated by $0.15 \mathrm{~mA}_{\mathrm{pp}}$, no rf excitation) versus $I_{\mathrm{dc}}$ at six different values of the in-plane magnetic field.

magnetic field, from $-8 \mathrm{~mA}$ at $0.81 \mathrm{kOe}$ to $-10 \mathrm{~mA}$ at $1.1 \mathrm{kOe}$ [see the continuous straight line in Fig. 4(d)], in agreement with the expected behavior of the threshold current Eq. (1). Hence, Fig. 4 presents a set of data consistent with the determination of a critical current of $I^{*}=-9 \mathrm{~mA}$ at $H_{0}=0.92 \mathrm{kOe}$, corresponding to $J_{c}^{*} \simeq 3 \times 10^{11} \mathrm{~A} \cdot \mathrm{m}^{-2}$, in agreement with the value of $2 \times 10^{11} \mathrm{~A} \cdot \mathrm{m}^{-2}$ expected from Eqs. (1) and (2) and the parameters of our system.

In conclusion, we have demonstrated that it is possible to fully control electronically the SW damping in a YIG microdisk. Nevertheless, the destabilization of dynamical modes is rather small, as the jump of the resonance field at $I^{*}$ (due to reduction of the magnetization) does not exceed the linewidth. We suspect that in our YIG|Pt microdisk, the splitting of modes is not sufficient to prevent nonlinear interactions that limit the amplitude of auto-oscillations [11]. In order to favor larger auto-oscillation amplitudes, YIG structures that are even more confined laterally (below $1 \mu \mathrm{m}$ ) should be used [29], or one should excite a bullet mode [13]. Extending this result to a one-dimensional SW guide [45] will offer a great prospect in the emerging field of magnonics [46,47], whose aim is to investigate the manipulation of SWs and their quanta, magnons.

This research was supported by the French Grants Trinidad (ASTRID 2012 program), by the RTRA Triangle de la Physique grant Spinoscopy, and by the Deutsche Forschungsgemeinschaft. S. O. D. and V. V. N. acknowledges respectively support from the Russian programs Megagrant No. 2013-220-04-329 and competitive growth of KFU. We acknowledge C. Deranlot, E. Jacquet, and R. Lebourgeois for their contribution to sample growth, and A. Fert for fruitful discussion. 
*Corresponding author.

oklein@cea.fr

† gregoire.deloubens@cea.fr

[1] S. O. Valenzuela and M. Tinkham, Nature (London) 442 , 176 (2006).

[2] T. Jungwirth, J. Wunderlich, and K. Olejnik, Nat. Mater. 11, 382 (2012).

[3] A. Manchon and S. Zhang, Phys. Rev. B 78, 212405 (2008).

[4] A. Thiaville, S. Rohart, É. Jué, V. Cros, and A. Fert, Europhys. Lett. 100, 57002 (2012).

[5] M. I. Dyakonov and V. I. Perel, JETP Lett. 13, 467 (1971).

[6] J. E. Hirsch, Phys. Rev. Lett. 83, 1834 (1999).

[7] I. M. Miron, K. Garello, G. Gaudin, P.-J. Zermatten, M. V. Costache, S. Auffret, S. Bandiera, B. Rodmacq, A. Schuhl, and P. Gambardella, Nature (London) 476, 189 (2011).

[8] L. Liu, C.-F. Pai, Y. Li, H. W. Tseng, D. C. Ralph, and R. A. Buhrman, Science 336, 555 (2012).

[9] K. Ando, S. Takahashi, K. Harii, K. Sasage, J. Ieda, S. Maekawa, and E. Saitoh, Phys. Rev. Lett. 101, 036601 (2008).

[10] L. Liu, T. Moriyama, D. C. Ralph, and R. A. Buhrman, Phys. Rev. Lett. 106, 036601 (2011).

[11] V. E. Demidov, S. Urazhdin, E. R. J. Edwards, M. D. Stiles, R. D. McMichael, and S. O. Demokritov, Phys. Rev. Lett. 107, 107204 (2011).

[12] K. An, D. R. Birt, C.-F. Pai, K. Olsson, D. C. Ralph, R. A. Buhrman, and X. Li, Phys. Rev. B 89, 140405 (2014).

[13] V. Demidov, S. Urazhdin, H. Ulrichs, V. Tiberkevich, A. Slavin, D. Baither, G. Schmitz, and S. O. Demokritov, Nat. Mater. 11, 1028 (2012).

[14] L. Liu, C.-F. Pai, D. C. Ralph, and R. A. Buhrman, Phys. Rev. Lett. 109, 186602 (2012).

[15] R. H. Liu, W. L. Lim, and S. Urazhdin, Phys. Rev. Lett. 110, 147601 (2013).

[16] Y. Kajiwara, K. Harii, S. Takahashi, J. Ohe, K. Uchida, M. Mizuguchi, H. Umezawa, H. Kawai, K. Ando, K. Takanashi, S. Maekawa, and E. Saitoh, Nature (London) 464, 262 (2010).

[17] C. W. Sandweg, Y. Kajiwara, K. Ando, E. Saitoh, and B. Hillebrands, Appl. Phys. Lett. 97, 252504 (2010).

[18] H. Kurebayashi, O. Dzyapko, V. E. Demidov, D. Fang, A. J. Ferguson, and S. O. Demokritov, Nat. Mater. 10, 660 (2011).

[19] L. H. Vilela-Leão, C. Salvador, A. Azevedo, and S. M. Rezende, Appl. Phys. Lett. 99, 102505 (2011).

[20] A. V. Chumak, A. A. Serga, M. B. Jungfleisch, R. Neb, D. A. Bozhko, V. S. Tiberkevich, and B. Hillebrands, Appl. Phys. Lett. 100, 082405 (2012).

[21] C. Hahn, G. de Loubens, O. Klein, M. Viret, V. V. Naletov, and J. Ben Youssef, Phys. Rev. B 87, 174417 (2013).

[22] O. d'Allivy Kelly, A. Anane, R. Bernard, J. Ben Youssef, C. Hahn, A. H. Molpeceres, C. Carretero, E. Jacquet, C. Deranlot, P. Bortolotti, R. Lebourgeois, J.-C. Mage, G. de Loubens, O. Klein, V. Cros, and A. Fert, Appl. Phys. Lett. 103, 082408 (2013).

[23] H. L. Wang, C. H. Du, Y. Pu, R. Adur, P. C. Hammel, and F. Y. Yang, Phys. Rev. B 88, 100406 (2013).

[24] Z. Wang, Y. Sun, M. Wu, V. Tiberkevich, and A. Slavin, Phys. Rev. Lett. 107, 146602 (2011).

[25] E. Padrón-Hernández, A. Azevedo, and S. M. Rezende, Appl. Phys. Lett. 99, 192511 (2011).

[26] Y.-T. Chen, S. Takahashi, H. Nakayama, M. Althammer, S. T. B. Goennenwein, E. Saitoh, and G. E. W. Bauer, Phys. Rev. B 87, 144411 (2013).
[27] M. B. Jungfleisch, T. An, K. Ando, Y. Kajiwara, K. Uchida, V. I. Vasyuchka, A. V. Chumak, A. A. Serga, E. Saitoh, and B. Hillebrands, Appl. Phys. Lett. 102, 062417 (2013).

[28] J. Xiao and G. E. W. Bauer, Phys. Rev. Lett. 108, 217204 (2012).

[29] C. Hahn, V. V. Naletov, G. de Loubens, O. Klein, O. d'Allivy Kelly, A. Anane, R. Bernard, E. Jacquet, P. Bortolotti, V. Cros, J. L. Prieto, and M. Muñoz, Appl. Phys. Lett. 104, 152410 (2014).

[30] Y. Sun, Y.-Y. Song, H. Chang, M. Kabatek, M. Jantz, W. Schneider, M. Wu, H. Schultheiss, and A. Hoffmann, Appl. Phys. Lett. 101, 152405 (2012).

[31] M. Althammer, S. Meyer, H. Nakayama, M. Schreier, S. Altmannshofer, M. Weiler, H. Huebl, S. Geprägs, M. Opel, R. Gross, D. Meier, C. Klewe, T. Kuschel, J.-M. Schmalhorst, G. Reiss, L. Shen, A. Gupta, Y.-T. Chen, G. E. W. Bauer, E. Saitoh, and S. T. B. Goennenwein, Phys. Rev. B 87, 224401 (2013).

[32] P. Pirro, T. Brächer, A. V. Chumak, B. Lägel, C. Dubs, O. Surzhenko, P. Görnert, B. Leven, and B. Hillebrands, Appl. Phys. Lett. 104, 012402 (2014).

[33] J.-C. Rojas-Sánchez, N. Reyren, P. Laczkowski, W. Savero, J.-P. Attané, C. Deranlot, M. Jamet, J.-M. George, L. Vila, and H. Jaffrès, Phys. Rev. Lett. 112, 106602 (2014).

[34] Z. Zhang, P. C. Hammel, and P. E. Wigen, Appl. Phys. Lett. 68, 2005 (1996).

[35] O. Klein, G. de Loubens, V. V. Naletov, F. Boust, T. Guillet, H. Hurdequint, A. Leksikov, A. N. Slavin, V. S. Tiberkevich, and N. Vukadinovic, Phys. Rev. B 78, 144410 (2008).

[36] H.-J. Chia, F. Guo, L. M. Belova, and R. D. McMichael, Phys. Rev. Lett. 108, 087206 (2012).

[37] Actually, the resonance peak shifts in field due to both the linear $(\approx 1.5 \mathrm{Oe} / \mathrm{mA})$ and quadratic $\left(\approx 0.5 \mathrm{Oe} / \mathrm{mA}^{2}\right)$ contributions in current of Oersted field and Joule heating, respectively.

[38] G. N. Kakazei, P. E. Wigen, K. Y. Guslienko, V. Novosad, A. N. Slavin, V. O. Golub, N. A. Lesnik, and Y. Otani, Appl. Phys. Lett. 85, 443 (2004).

[39] V. V. Naletov, G. de Loubens, G. Albuquerque, S. Borlenghi, V. Cros, G. Faini, J. Grollier, H. Hurdequint, N. Locatelli, B. Pigeau, A. N. Slavin, V. S. Tiberkevich, C. Ulysse, T. Valet, and O. Klein, Phys. Rev. B 84, 224423 (2011).

[40] Y. Tserkovnyak, A. Brataas, G. E. W. Bauer, and B. I. Halperin, Rev. Mod. Phys. 77, 1375 (2005).

[41] B. Heinrich, C. Burrowes, E. Montoya, B. Kardasz, E. Girt, Y.-Y. Song, Y. Sun, and M. Wu, Phys. Rev. Lett. 107, 066604 (2011).

[42] O. Mosendz, J. E. Pearson, F. Y. Fradin, G. E. W. Bauer, S. D. Bader, and A. Hoffmann, Phys. Rev. Lett. 104, 046601 (2010).

[43] V. Castel, N. Vlietstra, J. Ben Youssef, and B. J. van Wees, Appl. Phys. Lett. 101, 132414 (2012).

[44] A. Hamadeh, G. de Loubens, V. V. Naletov, J. Grollier, C. Ulysse, V. Cros, and O. Klein, Phys. Rev. B 85, 140408 (2012).

[45] Z. Duan, A. Smith, L. Yang, B. Youngblood, and I. N. Krivorotov, arXiv:1404.7262.

[46] V. V. Kruglyak, S. O. Demokritov, and D. Grundler, J. Phys. D 43, 264001 (2010).

[47] A. A. Serga, A. V. Chumak, and B. Hillebrands, J. Phys. D 43, 264002 (2010). 